\title{
Adenosine A1 receptor modifies P53 expression and apoptosis in breast cancer cell line Mcf-7
}

\author{
Nikbakht Dastjerdi $\mathrm{M}^{1}$, Valiani $\mathrm{A}^{2}$, Mardani $\mathrm{M}^{2}$, Zamani Ra Rani $\mathrm{M}^{2}$ \\ Department of Anatomical Sciences and central laboratory, Medical School, Isfahan University of Medical Sciences, \\ Isfahan, Iran.mz123esf@gmail.com
}

\begin{abstract}
BACKGROUND: Breast cancer cells over-express the adenosine receptor A1 and in most of these cells, P53 gene is a wild type. Because of this finding and relationship between A1 receptor and cell apoptosis and proliferation, this study aimed to determine the effect of agonist and antagonist of A1 receptor on cell apoptosis and proliferation and recognize the relationship between this receptor and P53 expression.

METHODS: We used a Real-Time PCR test for measuring expression of p53 gene also flow cytometry assay for apoptotic and survival cell rate after treatment of MCF-7 cells with A1 receptor agonist CPA (N6-Cyclopentyladenosine) and A1 receptor antagonist DPCPX (1,3-dipropyl-8-cyclopentylxanthine) in 24,48 and 72 hours. RESULTS: Our flow cytometry findings indicate that DPCPX significantly induces apoptosis in MCF-7. Also the expression of P53 becomes upregulated with time of DPCPX treatment. CPA treatment increased the survival cell rate and down-regulated this apoptosis-relevant gene P53 $(p>0.05)$.

CONCLUSION: DPCPX can induce P53 expression which consequently promotes the cell apoptosis in MCF7. Therefore, DPCPX could be used as an anti-cancer agent (Tab. 1, Fig. 3, Ref. 5). Text in PDF www.elis.sk. KEY WORDS: receptor, adenosine A1, apoptosis, gene, p53, MCF-7 cells.
\end{abstract}

\section{Introduction}

Breast cancer is the most frequent malignancy occurring in women in developed countries, where about one in eight women will have a lifetime risk of this cancer and one-third of patients will die of this disease $(1,2)$.

MCF-7 is one of the breast cancer cell lines that are seen in $70 \%$ of patients with breast cancer. In this cell line, P53 is a wild type (3)

Cancer cells use different mechanisms to escape from proliferation and growth control checkpoint while these mechanisms mainly accompany the genomic change in these cells. These changes can lead to abnormal cell proliferation, cell movement, and invasion or even increased drug resistance and other properties of malignant cells.

Changes in the cell signaling pathway can lead to these events and one of these changes is an expression of special receptors $(1,2)$.

A1 receptor is one of the Purinergic P1 receptors (A1, A2a, $\mathrm{A} 2 \mathrm{~b}$, and A3) that generally control immune and inflammatory reactions, apoptotic pathway, and cell division and differentiation. A1 receptor expression and concentration are different in different tissues and cells $(4,5,6,7)$.

${ }^{1}$ Department of Anatomical Sciences and central laboratory, Medical School, Isfahan University of Medical Sciences, Isfahan, Iran, and ${ }^{2}$ Department of Anatomical Sciences, Medical School, Isfahan University of Medical Sciences, Isfahan, Iran

Address for correspondence: M. Nikbakht Dastjerdi, Department of Anatomical Sciences and central laboratory, Medical School, Isfahan University of Medical Sciences, Isfahan, Iran.

Phone/Fax: +98.31.36700477
Programmed cell death or apoptosis is used for various biological events such as senescence, clearance of excess and harmful cells. This type of death is essential for normal development and homeostasis. Two main apoptotic pathways consist of extrinsic or death receptor-mediated apoptosis and intrinsic or mitochondriamediated apoptosis (8). Many drugs used for cancer treatment induce apoptotic death through the p53-dependent pathway (9).

P53 is one of the important factors for controlling mitosis and cell proliferation; also well known as a tumor suppressor gene in human genome. In cancers, P53 is a reliable biomarker to determine the prognoses of patients and follow-up treatment results. This molecule has several biological functions including DNA repair, cell cycle control, differentiation, and apoptosis. P53 is mutated in $50 \%$ of human cancers but this rate in breast cancer is only $20-30 \%$, and in most cases this gene is a wild type. In addition, P53 is a key initiator in external and internal apoptotic pathways. Inhibition of P53 gene expression can also cause uncontrolled cell division $(8,10)$.

A1 receptor is expressed in all types of breast cancer cells but so far the signaling pathway of A1 receptor in these cells is not clear (11). Some researchers showed that A1 receptor expression increased the cell viability and mitosis rate and decreased the apoptosis in these cells. This expression is critical also for estrogen receptor function and signaling $(12,11)$.

Studies show that the activation of A3 receptor with IB-MECA as an agonist for this receptor in prostate cancer leads to P53 overexpression, cell cycle arrest, and caspase-3 activation (13). In the kidney, brain and heart, the activation of A1 receptor reduced cell damage and apoptotic rate following oxidative stress while the 
antagonist of this receptor increased cell and tissue damage under these conditions (14). This receptor can inhibit caspase-3 activation as well as apoptosis in hypoglycemic brain (15).

In this study we investigated the effects of A1 receptor agonist (CPA, N6-Cyclopentyladenosine) and antagonist (DPCPX, 8-Cyclopentyl-1, 3-dipropylxanthine) on expression of P53 and apoptotic rate.

\section{Materials and methods}

\section{Cell lines, drug, treatment and culture condition}

Mcf-7 cell line obtained from the Pastor Institute of Animals Cell Culture. Cells were maintained in Dulbecco's modified Eagle medium (DMEM/F12) with $10 \% \mathrm{FBS}, 100 \mathrm{U} / \mathrm{ml}$ penicillin, 100 $\mu \mathrm{g} / \mathrm{ml}$ streptomycin and kept at $37^{\circ} \mathrm{C}$ in a humidified atmosphere with $5 \% \mathrm{CO}_{2}$. For experiments after trypsinization (Trypsin-EDTA), cells were seeded in culture flasks. CPA and DPCPX was purchased from Sigma (C8031, C101) and dissolved in DMSO to the appropriate concentrations according to IC50 assay. After the cells had been $>80 \%$ confluent and grown exponentially in $75 \mathrm{~mm}$ diameter culture flask, $10^{6} \mathrm{MCF}-7$ cells were counted and plated in 75-mm diameter culture flask and kept in culture medium for 24 hours which were then incubated with certain concentrations of CPA and DPCPX, based on IC50 index for $24 \mathrm{~h}$ and at certain times $(24,48$ and $72 \mathrm{~h})$ the tests were performed.

\section{IC50 assay}

The IC50 values for CPA and DPCPX in MCF-7 groups were acquired after 24 hours of treatment. Briefly, $10^{4}$ cells (MCF-7) were counted and placed into each well of a 12-well plate and were treated with various drug concentrations $(0,10,50,100,200,400$ $\mu \mathrm{M}$ doses for CPA and $10 \mathrm{nM}, 50 \mathrm{nM}, 100 \mathrm{nM}, 1 \mu \mathrm{M}, 10 \mu \mathrm{M}$, $100 \mu \mathrm{M}$ doses for DPCPX) for 24 hours, and the MTT survival assay was then carried out for evaluating the cell viability with dif-
Tab. 1. Primers used in real-time PCR.

\begin{tabular}{lll}
\hline 1 & GAPDH forward & CACCACCATGGAGAAGGCTGG \\
\hline 2 & GAPDH reverse & CCAAAGTTGTCATGGATGACC \\
\hline 3 & P53 forward & CGAGTCCCGCGGTAATTCTT \\
\hline 4 & P53 reverse & TGCAGAAGAGGTGCAAGACC \\
\hline
\end{tabular}

ferent drug concentrations in each groups. A graph of cell viability versus drug concentration was used to calculate IC50 values for MCF-7 cell line.

\section{Real-Time PCR}

Total RNA from cells was extracted at the indicated times, using the RNeasy mini kit and following the instructions from the manufacturer (Qiagen, Hilden, Germany). After treatment with DNase I, Total RNA (100 ng) was reverse-transcribed to cDNA by using the RevertAid ${ }^{\mathrm{TM}}$ First Strand cDNA Synthesis Kit (Fermentas) according to the manufacturer's instructions. The Maxima SYBR Green Rox qPCR master mix kit (Fermentas) was used for real-time RT-PCR. Primer sequences are shown in Table 1. Realtime PCR reactions were performed with StepOnePlus (Applied Biosystem). The program of Real-Time PCR consisted of $10 \mathrm{~min}$ at $95{ }^{\circ} \mathrm{C}$ followed by 40 cycles of denaturation step at $95{ }^{\circ} \mathrm{C}$ for $15 \mathrm{sec}$ following by annealing and extension for $1 \mathrm{~min}$ at $60^{\circ} \mathrm{C}$. Data were analyzed by Comparative $\mathrm{Ct}(\Delta \Delta \mathrm{ct})$ method. The relative expression level of p53 was calculated by determining the ratio between the amount of $\mathrm{p} 53$ and that of endogenous control. Melting curve analysis $\left(60^{\circ} \mathrm{C} \rightarrow 95^{\circ} \mathrm{C}\right.$ increment of $\left.0.3^{\circ} \mathrm{C}\right)$ was used to determine the melting temperature of specific amplification products and primer dimers. These experiments were carried out in triplicate and were independently repeated at least 3 times.

\section{Flow-Cytometric analysis}

The percentage of apoptotic cells was measured by flow cytometry following AnnexinV (FL1-H) and PI (FL2-H) labeling.
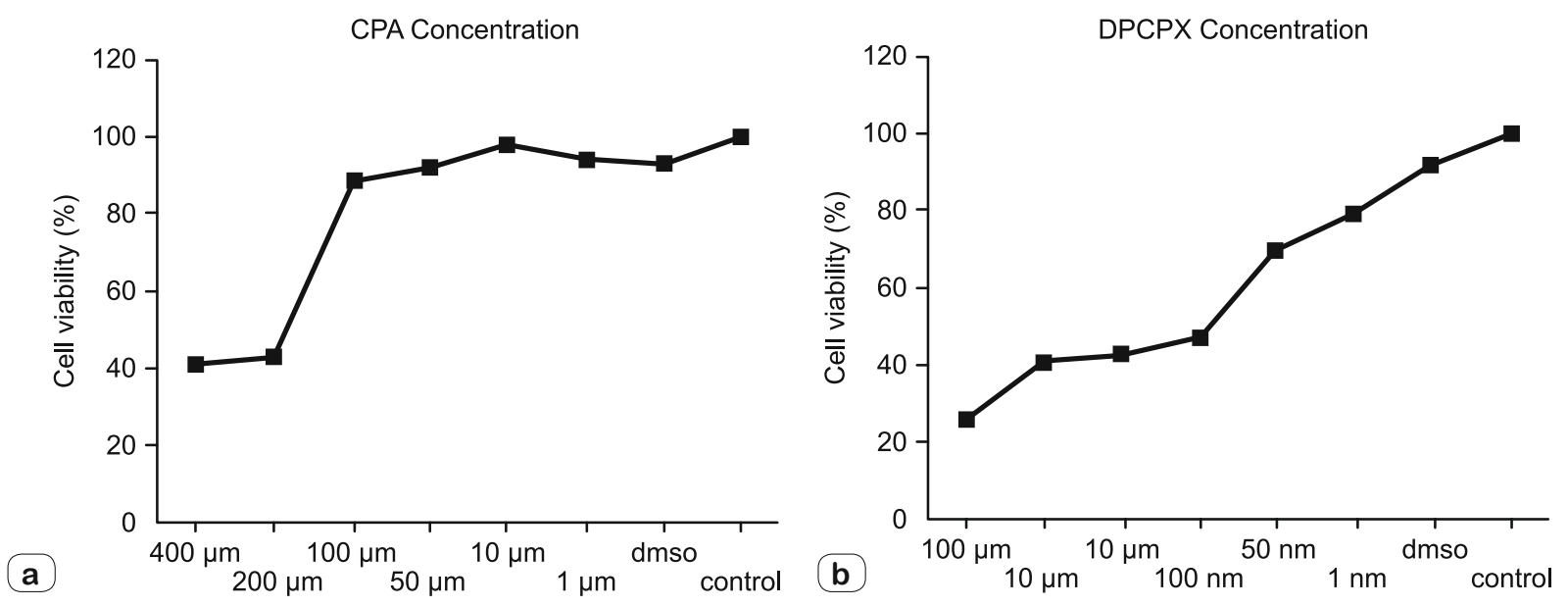

Fig. 1a, b. IC50 assay for half maximal inhibitory concentration analysis of CPA (1a) and DPCPX (1b) in MCF7 cancer cell lines after $24 \mathrm{~h}$ of treatment. Cells were incubated with/or without the drug in different concentrations, and the relative amount of viable cells were estimated by measuring the absorbance of the cell suspension after incubation with MTT assay Graph of viability versus drug concentration was used to calculate IC50 values. 


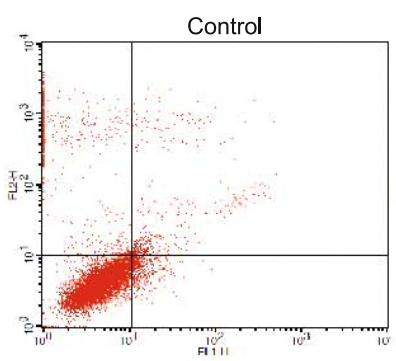

DPCPX $24 \mathrm{~h}$

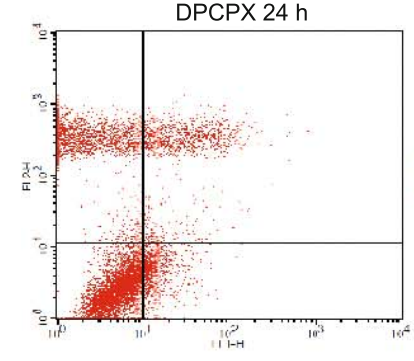

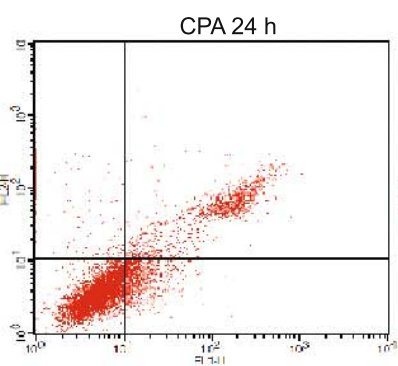

DPCPX $48 \mathrm{~h}$

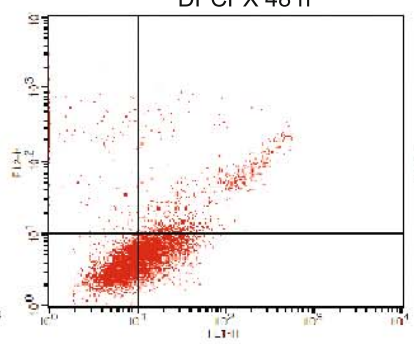

CPA $48 \mathrm{~h}$

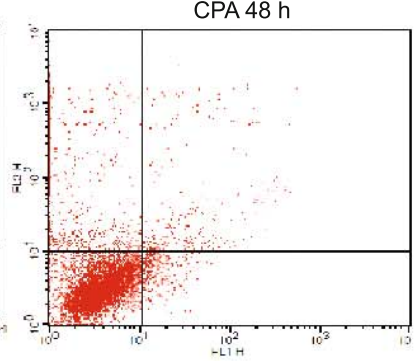

DPCPX $72 \mathrm{~h}$

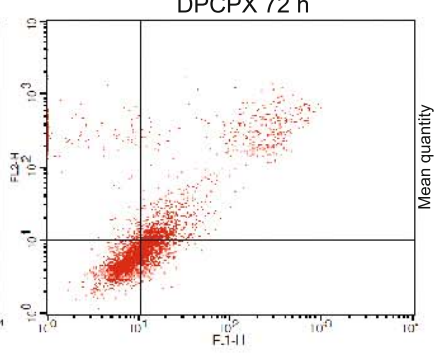

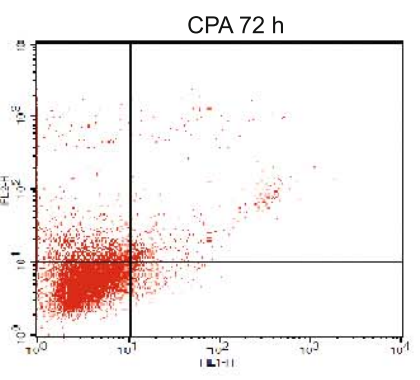

Result analyzis

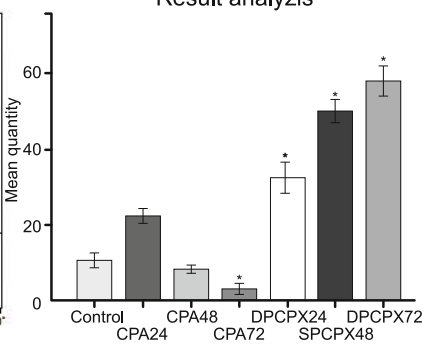

Fig. 2. DPCPX induces apoptosis in cancer cells line (MCF-7) but CPA does not have this manner and it also haa an anti-apoptotic effect. Relative levels of apoptotic cells in MCF-7 cancer cell lines treated with $87 \mathrm{nM}$ DPCPX and $180 \mu \mathrm{M}$ CPA for different times. The percentage of apoptotic cells was measured using the AnnexinV FITC and PI assay as described previously. Cells that are AnnexinV-positive and propidium iodide-negative are in early apoptosis, as phosphatidyl serine (PS) translocation has occurred, although the plasma membrane remains intact. Cells that are positive for both AnnexinV and PI either are in the late stages of apoptosis or are already dead, as PS translocation has occurred, and the loss of plasma membrane integrity is visible.

A minimum of $4 \times 10^{5}$ cells/ $\mathrm{ml}$ were analyzed for each sample. Cells were treated with CPA and DPCPX $(180 \mu \mathrm{M}$ and $87 \mathrm{nM})$ for 24 and then in 24, 48, and 72 hours washed in PBS and resuspended in binding buffer $(1 \times ; 5 \mu \mathrm{l})$. AnnexinV-FITC was added to $195 \mu \mathrm{l}$ cell suspensions and then the analysis was carried out according to the manufacturer's protocol (BMS500F1/100CE AnnexinV-FITC, eBioscience, USA). Finally, the apoptotic cells were counted by FACScan flow cytometry (Becton Dickinson, Heidelberg, Germany). These experiments were carried out in triplicate and were independently repeated at least 3 times.

\section{Statistical analysis}

All the quantitative data were presented as the mean \pm standard deviation. One-way analysis of variance (ANOVA) with LSD post-hoc test was performed to determine statistical significance among different groups by using SPSS software package 16.0. Significance was accepted at a level of $\mathrm{p}<0.05$.

\section{Results}

\section{Results of IC50 assay}

After the treatment of MCF-7 cells with MTT solution, the dark blue formazan crystals were seen in viable cells, which indicated their metabolic activity. The reduction in the number of cells was dependent on the cell types as shown by the half-maximal inhibitory concentration (IC50) index. Then the IC50 values for the CPA and DPCPX were established (Fig. 1a). The results show the essential concentration of CPA while DPCPX to achieve the IC50 in MCF-7 cells at 24 hours was $180 \mu \mathrm{M}$ and $87 \mathrm{nM}$ (Fig. 1b).

\section{Results of Flow cytometry}

The flow cytometry assay was used to determine the apoptotic potential of the CPA and DPCPX; these results showed that the $87 \mathrm{nM}$ concentration of DPCPX based on IC50 index at different times $(24,48$ and $72 \mathrm{~h})$ could significantly induce apoptosis in MCF-7 cells while it increased with elapsing time $(\mathrm{p}<0.05)$ (Fig. 2). DPCPX treatment arrested MCF-7 cell proliferation and induced apoptosis ( $\geq 65 \%$ of inhibition) in 72 hours, whereas the effect of CPA on cells' programmed death at all different times was negative ( $p>0.05)$ (Fig. 2). MCF-7 apoptotic cells showed a sharp increase at all times in comparison with control group cells $(\mathrm{p}<0.05)$. DMSO was used in the control sample (drug vehicle) and a small amount of cell apoptosis in cell line at different times was observed $(\mathrm{p}<0.05)$ (Fig. 2).

\section{Results of Real-Time PCR}

To examine CPA and DPCPX effects (based on IC50 index) at different times on the mRNA expression of P53 gene on MCF-7 cells we used Real-Time quantitative PCR. The P53 gene expression was dramatically up-regulated by DPCPX treatment with elapsing time in MCF-7 cells, in particular at 72 hours of treatment its expression was significantly raised (Fig. 3) $(p<0.05)$. CPA significantly down-regulated the genes' expression at different times ( $p>0.05)$, especially at 72 hours (Fig. 3).

\section{Discussion}

Recently, several groups have reported the use of cell receptors such as adenosine receptors family for successful controlling or 


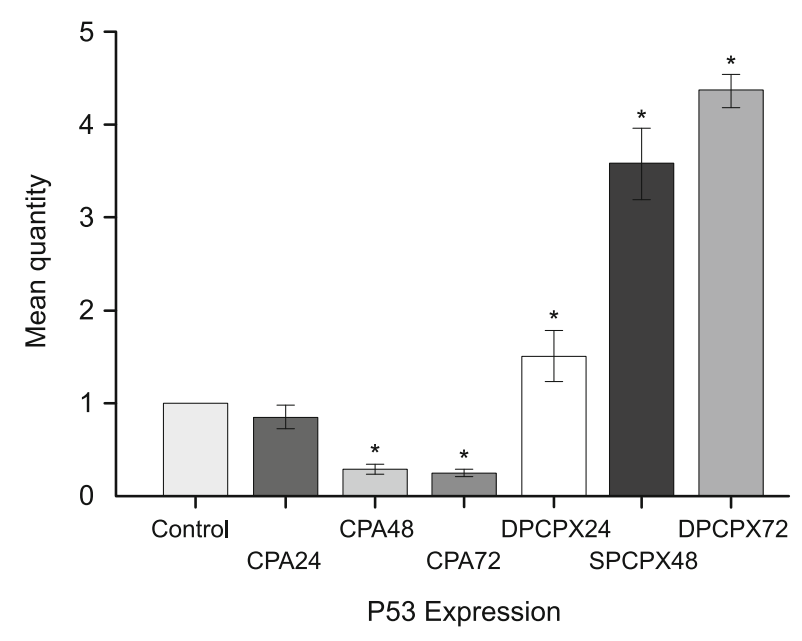

Fig. 3. Effects of CPA and DPCPX on the Level of p53 expression in MCF-7 cells in 24, 48, and $72 \mathrm{~h}$ after treatment. Cells were cultured as described in Materials and methods.

overcoming resistance to cancer drugs (7). In cancer therapy, the ideal therapeutic approach would be to induce apoptosis specifically to primary tumors, as well as those at the site of metastatic cells while simultaneously monitoring the prognosis through noninvasive approaches (16).

Adenosine A1 receptor is a member of the G protein-coupled receptor (GPCR) super family. Based on different roles of adenosine A1receptor in some diseases, it has also been suggested that this receptor may act as a potent regulator of the growth of normal and tumor cells by exerting pro- and anti-apoptotic or pro-survival effects $(17,18,19)$. It was suggested earlier that apoptotic induction in cancer cell lines by adenosine receptor A1 antagonist requires the activation of P53 gene expression $(2,10)$. In this study, responses of MCF-7 cells against agonist and antagonist of A1 receptor were investigated.

Our results in flow cytometry assay demonstrated that the use of adenosine A1 receptor antagonist (DPCPX) induced apoptosis in MCF-7 cells and adenosine A1 receptor agonist (CPA) decreased apoptosis rate. MTT assays revealed that DPCPX exerts significant cytotoxicity to MCF-7 cells in time-dependent fashion in the concentration of $87 \mathrm{nM}$. We analyzed the mRNA expression levels of p53 gene in response to DPCPX and CPA exposure in MCF-7 cells, because two main apoptosis pathways are controlled through these molecules. Quantitative Real-Time PCR results showed that DPCPX up-regulated mRNA level of cell cycle checkpoint protein p53. Expression of these genes was down-regulated in cells treated with CPA. Taken together, up-regulation of P53 leads to activation of pro-apoptotic members of bcl-2 family such as Bax which induces permeabilization of the outer mitochondrial membrane, which in turn releases soluble proteins from the intermembrane space into the cytosol that can induce caspase activation while this activation starts the final apoptosis $(19,20)$.

Previous studies show that adenosine induces $\mathrm{HuH}-7$ cell apoptosis in a caspase-dependent manner (21) and A1 adenosine receptor plays a critical role in the development of glioblastomas.
This role is mediated by microglia cells (22). But so far, no evidence has been reported that $\mathrm{A} 1$ adenosine receptors induce P53 expression and apoptosis, conversely, it has been shown that these receptors decrease apoptotic cell death in some cells $(23,24)$. Our findings oppose K. Sai et al results from astrosytoma RCR-1 cell line which show that $\mathrm{A} 1$ adenosine receptor agonist (CHA) induces apoptosis by activating caspase- 9 and the ensuing caspase- 3 via being linked to A1 adenosine receptor in RCR-1 cells (25).

In cancer, targeted therapy is one of the key challenges that make use of agents specific for the deregulated proteins of cancer cells in each site of body such as specific receptors and generally deactivate the enzymatic domains on mutated, over-expressed, or otherwise critical proteins within the cancer cell (16) Therefore special cell receptors have the potential to play a key role in achieving such goals. It is anticipated that $\mathrm{A} 1$ receptor antagonist might significantly reduce the usage of anti-cancer drugs with better specificity, enhanced efficacy and lower toxicities and side effects because it is targeted on special cells only.

In conclusion, we have shown that $\mathrm{A} 1$ receptor antagonist (DPCPX) produce significant apoptosis in MCF-7 cells in the concentration range of $180 \mathrm{nM}$. Furthermore, quantitative Real-Time PCR analysis displayed that mRNA level of P53 involved in the apoptosis was altered by this treatment. Overall, our data suggest that A1 receptor antagonist may induce apoptosis in MCF-7 cells via 553 over-expression. This in vitro study showing the induction of apoptosis by A1 receptor antagonist warrants further investigation to determine whether in vivo treatment consequences may exist for this receptor application.

\section{References}

1. Osborne C, Wilson P, Tripathy D. Oncogenes and tumor suppressor genes in breast cancer: potential diagnostic and therapeutic applications. The Oncologist 2004; 9: 361-377.

2. Adair TH. Growth regulation of the vascular system: an emerging role for adenosine. J Physiol Regul Integr Comp Physiol 2005; 289: 283-296.

3. Holliday DL, Speirs V. Choosing the right cell line for breast cancer research. Breast Cancer Res 2011; 13: 215.

4. Williams M, Jarvis MF. Purinergic and Pyrimidinergic Receptors as Potential Drug Targets. Biochem Pharmacol 2000; 59: 1173-1185.

5. Burnstock G. Pathophysiology and Therapeutic Potential of Purinergic Signaling. Pharmacol Rev 2006; 58: 58-86.

6. Burnstock G. Purine and pyrimidine receptors. Cell Mol Life Sci 2007; 64 (12): 1471-1483.

7. Merighia S, Mirandolab P, Varani K et al. A glance at adenosine receptors: novel target for antitumor therapy. Pharmacol Ther 2003; 100: 31-48.

8. Rajesh P, Rastogi R, Sinha RP. Apoptosis: Molecular Mechanisms and Pathogenicity. EXCLI J 2009; 8: 155-181.

9. Elmore S. Apoptosis: A Review of Programmed Cell Death. Toxicol Pathol 2007; 35 (4): 495-516.

10. Fridman JS, Lowe SW. Control of apoptosis by p53. Oncogene 2003; 22: $9030-9040$.

11. Mirza A, Basso A, Black $\mathbf{S}$ et al. RNA Interference Targeting of $A 1$ Receptor-Over expressing Breast Carcinoma Cells Leads To Diminished 
242-246

Rates of Cell Proliferation and Induction of Apoptosis. Cancer Biol Ther 2005; 4: 12, 1355-1360.

12. Lin ZH, Yin P, Reierstad S et al. Adenosine A1 receptor, a target and regulator of $\mathrm{ER} \alpha$ action, mediates the proliferative effects of estradiol in breast cancer. Oncogene 2010; 8: 1114-1122.

13. Aghaei M, Panjehpour M, Karami-Tehrani F et al. Molecular mechanisms of A3 adenosine receptor-induced G1 cell cycle arrest and apoptosis in androgen-dependent and independent prostate cancer cell lines: involvement of intrinsic pathway. J Cancer Res Clin Oncol 2011; 137: 1511-1523.

14. Lee HT, Gallos G, Nasr SH et al. A1 Adenosine Receptor Activation Inhibits Inflammation, Necrosis, and Apoptosis after Renal Ischemia-Reperfusion Injury in Mice. J Am Soc Nephrol 2004; 15: 102-111.

15. Turner CP, Blackburn MR, Rivkees SA. A1 adenosine receptors mediate hypoglycemia-induced neuronal injury. J Mol Endocrinol 2004; 32: $129-144$.

16. Selim ME, Hendi AA. Gold Nanoparticles Induce Apoptosis in MCF7 Human Breast Cancer Cells. Asian Pacific J Cancer Prev 2012; 13: $1617-$ 1620 .

17. Ma' laga-Die' guez L, Yang Q, Bauer J et al. Pharmacochaperoning of the A1 Adenosine Receptor Is Contingent on the Endoplasmic Reticulum. Mol Pharmacol 2010; 77: 940-952.

18. Aljarari NMH, Sheriff DS. A Pharmacological Characterization of Adenosine Receptors. Drug discovery 2012; 2 (5): 165-170.
19. Kanduc D, Mittelman A, Serpico R et al. Cell death: Apoptosis versus necrosis. Int J Oncol 2002; 21 (1): 165-170.

20. Qin JZ, Stennett L, Bacon P et al. Resistance of malignant melanomas p53-independent NOXA induction overcomes apoptotic. Mol Cancer Ther 2004; 3: 895-902.

21. Yang D, Yaguchi T, Nakano T, Nishizaki T. Adenosine-induced caspase- 3 activation by tuning Bcl-XL/DIABLO/IAP expression in HuH-7 human hepatoma cells. Cell Biol Toxicol 2010; 26: 319-330.

22. Synowitz M, Glass R, Färber K et al. A1 Adenosine Receptors in Microglia Control Glioblastoma-Host Interaction. Cancer Res 2006; 66: $8550-8557$.

23. Lee HT, Gallos G, Nasr SH, Emala CW. A1 adenosine receptor activation inhibits inflammation, necrosis, and apoptosis after renal ischemiareperfusion injury in mice. J Am Soc Nephrol 2004; 15: 102-11.

24. Regan SE, Broad M, Byford AM et al. A1 adenosine receptor overexpression attenuates ischemia reperfusion- induced apoptosis and caspase 3 activity. Am J Physiol Heart Circulat Physiol 2003; 284: H859-866.

25. Katsunari Sai, Dongqin Yang, Hideyuki Yamamoto. A1 adenosine receptor signal and AMPK involving caspase-9/-3 activation are responsible for adenosine-induced RCR-1 astrocytoma cell death. NeuroToxicol 2006; $27: 458-467$.

Received June 13, 2015. Accepted September 15, 2015. 